\title{
Rheology of wet granular materials under continuous shear: experiments and simulations
}

\author{
Michel Badetti ${ }^{1, \star}$, Abdoulaye Fall ${ }^{1, \star \star}$, and Jean-Noël Roux ${ }^{1, \star \star \star}$ \\ ${ }^{1}$ Laboratoire Navier, UMR8205, CNRS-ENPC-IFSTTAR,2 allée Kepler, 77420, Champs-sur-Marne, France
}

\begin{abstract}
The behaviour of wet granular media in shear flow is characterized by the dependence of apparent friction $\mu^{*}$ and solid fraction $\Phi_{S}$ on the reduced pressure $P^{*}$ and the inertia number $I$. Reduced pressure, $P^{*}=\sigma_{22} a^{2} / F_{0}$, compares the applied normal stress $\sigma_{22}$ on grains of diameter $a$ to the tensile strength of contact $F_{0}$ (proportional to the surface tension $\Gamma$ of the liquid and the beads diameter). A specifically modified rotational rheometer is used to characterize the response of model wet granular material to applied shear rate $\dot{\gamma}$ under controlled normal stress $\sigma_{22}$. Discrete Element Method (DEM) simulations in 3D are carried out in parallel and numerical results are compared with experimental ones. Cohesive, inertia, saturation and viscous effects on macroscopic coefficient of friction $\mu^{*}$ and solid fraction $\Phi_{S}$ are discussed.
\end{abstract}

\section{Introduction}

In the steady state, shear flows of granular materials under controlled normal stress $\sigma_{22}$ and shear rate $\dot{\gamma}$ (see Fig. 1) are well described by the solid fraction $\Phi_{S}$ and the macroscopic friction coefficient $\mu^{*}=\sigma_{12} / \sigma_{22}$. Characterizing flow inertia of such materials with the inertial number $I=\dot{\gamma} a \sqrt{m / a \sigma_{22}}$ ( $m$ being the mass of a grain and $a$ its diameter) is now a classical approach $[1,2]$. However, recent studies showed that in the case of wet granular materials there is also a strong dependency of both $\mu^{*}$ and $\Phi_{S}$ on the cohesive effect induced by liquid bonding between particles [3-5]. For a pack of beads of diameter $a$ with bonds strength $f_{0}$, the reduced pressure $P^{*}=a^{2} \sigma_{22} / f_{0}$ can characterize this cohesive effect.

Previous DEM studies proposed constitutive laws $\mu^{*}\left(I, P^{*}\right)$ and $\Phi_{S}\left(I, P^{*}\right)$ covering broad ranges of $I$ and $P^{*}$ [3]. The aim of the present commuication is to compare these DEM simulations of wet grain assemblies with new experimental measurements using a normal stress controlled shear cell, both in the quasistatic limit and in dense inertial flows.

Sec. 2 describes the model material and the shearing methods. Sec. 3.1 compares flow curves of our materials obtained numerically and experimentally, in Sec. 3.4 and 3.5 respectively the influence of liquid volume $\Phi_{L}$ and viscosity $\eta$ in the quasistatic regime is discussed.

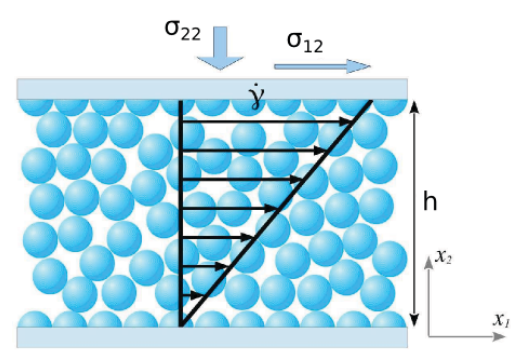

Figure 1. Ideal plane shear with confinement. shear rate is defined as $\dot{\gamma}=\partial \dot{x_{1}} / \partial x_{2}$.

\section{Methods}

\subsection{Model material}

In simulations and experiments, the model material proposed is a simple pack of monodisperse spherical polystyrene beads of diameter $a=5 \times 10^{-4} \mathrm{~m}$ mixed together with a newtonian silicon oil of surface tension $\Gamma=47 \mathrm{mN} \cdot \mathrm{m}^{-1}$ and variable viscosity $\eta$ ranging from $20 \mathrm{mPa}$.s up to $1000 \mathrm{mPa}$.s. Liquid quantity is described by the ratio $\Phi_{L} / \Phi_{S}$ which ranges from 0.003 up to 0.075 [6-8], thus ensuring a pendular state. In such state, for a perfecting wetting fluid bond strength at contact $f_{0}$ is well approximated by $\pi a \Gamma=7.38 \times 10^{-5} \mathrm{~N}[9]$.

\footnotetext{
$\star$ e-mail: michel.badetti@ifsttar.fr

$\star \star$ e-mail: abdoulaye.fall@ifsttar.fr

$\star \star \star$ e-mail: jean-noel.roux @ifsttar.fr
} 


\subsection{Experimental setup}

Materials are inserted in a specifically devised rheological cell (see Fig. 2). Constant force $F_{N}$ and rotation rate $w$ are applied on a volume $V_{S}$ of material, Torque $T$ and material width $h$ (see Fig. 1) are measured once plateau values of the steady state are reached. Assuming plane shear with truncated center, $\sigma_{12}, \sigma_{22}, \dot{\gamma}$ and $\Phi_{S}$ are calculated as :

$$
\begin{aligned}
\sigma_{12} & =\frac{3 T}{2 \pi R_{e}^{3}\left(1-\left(R_{i} / R_{e}\right)^{3}\right)} \\
\sigma_{22} & =\frac{F_{N}}{\pi R_{e}^{2}\left(1-\left(R_{i} / R_{e}\right)^{2}\right)} \\
\dot{\gamma} & =w \frac{R_{e}+R_{i}}{2 h} \\
\Phi_{S} & =\frac{V_{S}}{\pi h R_{e}^{2}\left(1-\left(R_{i} / R_{e}\right)^{2}\right)}
\end{aligned}
$$

Materials are inserted to ensure a width $h>20 a$, the gap between inner $R_{i}$ and outer radii $R_{e}$ is equal to $20 a$. Experimental measures with different values of $h$ (see figure 3 showed no gap effect ruling out possibilities of localization effects. Similar devices have already been used to describe succesfully the rheology of dense suspensions and dry materials $[1,2,10]$. Means and error bars are obtained for materials tested at least 3 times.

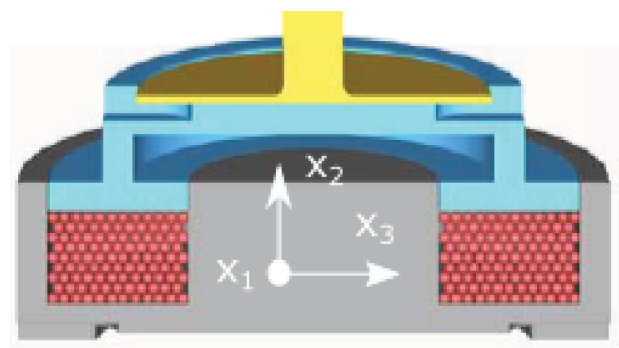

Figure 2. Rheological cell : gains (red) are trapped between the stator (grey) and the rotor (blue+yellow). Shear happens in the $\mathbf{x}_{1}, \mathbf{x}_{2}$ plane. Outer and inner radii are respectively $R_{e}$ and $R_{i}$

\subsection{Numerical simulations}

Materials are placed in a 3D periodical cell sheared using Lees-Edwards boundary conditions. Shear rate $\dot{\gamma}$ and $\sigma_{22}$ are applied, $\sigma_{12}$ and $\Phi_{S}$ are measured. Contact interaction are implemented using an Hertz-Mindlin model with viscous dissipation, friction in contact satisfies Coulomb's law with a friction coefficient $\mu=0.09$ chosen in order to fit values of $\mu^{*}=\mu_{d}^{*}=0.257$ and $\Phi_{S}=\Phi_{S, d}=0.616$ for $I \rightarrow 0$ and $P^{*} \rightarrow \infty$ found experimentally (see figure 4). Distant interactions involve meniscii assumed to form only when grain come into contact with a fixed volume $V_{m}$, they disappear at a certain rupture distance $D_{0}=V_{m}^{1 / 3}$. Error bars are obtained using the technique of block transform estimates [11].
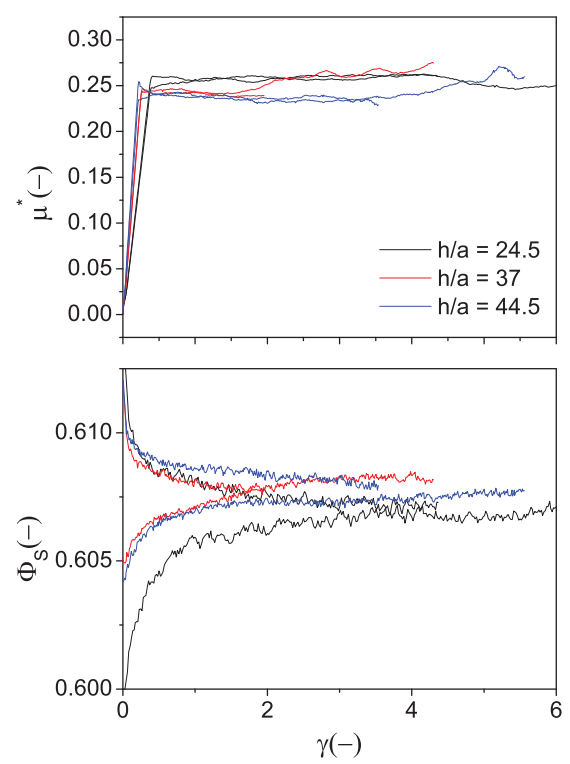

Figure 3. $\mu^{*}$ and $\Phi_{S}$ measured at different sample width. Absence of gap effect is highlighted. Measurements made with $\eta=20 \mathrm{mPa} . \mathrm{s}, \Phi_{L} / \Phi_{S}=0.075, I=10^{-4}$ and $P^{*}=3$

\section{Results}

\subsection{Inertia and cohesive effects}

Figure 4 presents $\mu^{*}$ and $\Phi_{S}$ as functions of $I$ for different values of $P^{*}$ in experiments and simulations. For low values of $\mathrm{I}\left(<10^{-3}\right)$, ie in the quasistatic regime, $\mu^{*}$ and $\Phi_{S}$ reach plateau values. For higher values of $I$, in the inertial regime, $\mu^{*}$ increases and $\Phi_{S}$ decreases upon increasing $I$.

Table 1 compares experimental and numerical data when $I=10^{-3}$, values recorded with the two methods show very good agreements with less than $6 \%$ relative difference for $\mu^{*}$ and less than $3 \%$ relative difference for $\Phi_{S}$.

The agreement between simulations and experiments remains good when comparing the solid fractions for inertial flows, the maximal relative difference between experiments and simulations is at most $0.5 \%$ for $P^{*}=2$ and $0.8 \%$ for $P^{*}=3$. There is, however, a discrepancy when looking at the values of $\mu^{*}$ in the inertial regime. When $I>3 \cdot 10^{-3}$, the relative difference rises up to $30 \%$ for $\mathrm{P}^{*}=3$ and up to $17 \%$ for $\mathrm{P}^{*}=2$. Clearly, $\mu^{*}$ increases much faster with $I$ away from its quasistatic value $\mu_{0}^{*}$ for $I \rightarrow 0$ in the experiments than in the numerical simulations. This discrepancy could emerge from questions of liquid repartition in the experimental samples.

Table 1. Values of $\mu_{0}^{*}$ and $\Phi_{S}$ obtained for $I<10^{-3}$, $\Phi_{L} / \Phi_{S}=0.03$ and $\eta=550 \mathrm{mPa}$.s. exp and num subscripts respectively stand for experimental and numerical

\begin{tabular}{lllll}
\hline$P^{*}$ & $\mu_{\text {exp }}^{*}$ & $\mu_{\text {num }}^{*}$ & $\Phi_{S, \exp }$ & $\Phi_{S, \text { num }}$ \\
\hline$\infty$ & 0.255 & 0.257 & 0.616 & 0.616 \\
3 & 0.302 & 0.327 & 0.606 & 0.605 \\
2 & 0.340 & 0.364 & 0.595 & 0.597 \\
\hline
\end{tabular}




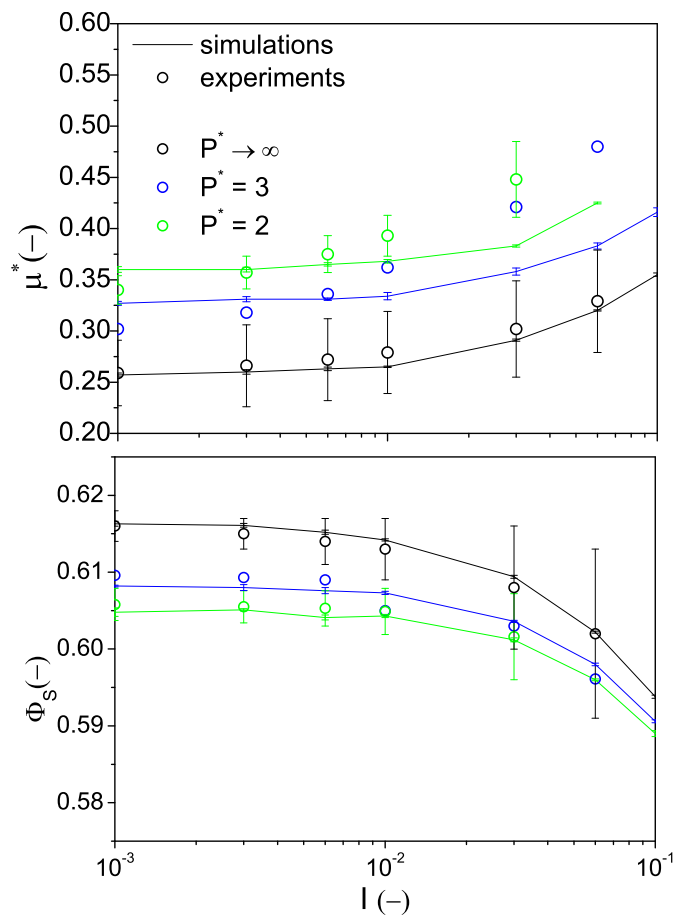

Figure 4. Flow curves $\mu^{*}\left(I, P^{*}\right)$ and $\Phi_{S}\left(I, P^{*}\right)$. filled circles represent experimental data while lines representnumerical data. Dry samples in black, $P^{*}=3$ in blue and $P^{*}=2$ in green. For wet samples viscosity $\eta=550 \mathrm{mPa}$.s and $\Phi_{L} / \Phi_{S}=0.015$.

\subsection{Quasistatic regime : Saturation effect}

Fig. 5 shows numerical and experimental $\mu_{0}^{*}$ versus $1 / P^{*}$ for different ratio $\Phi_{L} / \Phi_{S}$. For poorly cohesive materials $\left(1 / P^{*}=0.033\right)$, numerically increasing $\Phi_{L} / \Phi_{S}$ from 0.015 up to $0.06, \mu_{0}^{*}$ grows by $1 \%$. For highly cohesive ones $\left(1 / P^{*}=1\right)$, increasing $\Phi_{L} / \Phi_{S}$ from 0.015 up to 0.075 experimentally and from 0.015 up to $0.06, \mu_{0}^{*}$ grows by $16 \%$. On the whole range of $1 / P^{*}$, Fig. 5 also shows that $\Phi_{S}$ barely depends on $\Phi_{L} / \Phi_{S}$.

Assuming the quasistatic behavior of wet granular materials can be expressed through a Mohr-Coulomb criterion, e.g materials flow with :

$$
\sigma_{12}=\mu_{d}^{*} \sigma_{22}+C
$$

where cohesion $C$ is defined as a rupture tangential stress in unconfined conditions. Ensuring $\sigma_{22}=0 \mathrm{~Pa}$ is not possible with the methods at our disposal. However, knowing the constant macroscopic coefficient of friction $\mu_{d}^{*}$ of dry granular material, $C$ can be estimated at various $\sigma_{22}$. Using equation 1 , numerical estimation are averaged for $\sigma_{22}=9000 \mathrm{~Pa}$ and $4500 \mathrm{~Pa}$, experimental ones are averaged for $\sigma_{22}=1500 \mathrm{~Pa}$ and $1200 \mathrm{~Pa}$, the values obtained are reported in table 2. Estimates of $C$ increase up to $39 \%$ within the range of $\Phi_{L} / \Phi_{S}$. Noting that Eq. 1 is equivalent to :

$$
\mu_{0}^{*}=\mu_{d}^{*}+\frac{a^{2} C}{f_{O}} \frac{1}{P^{*}}
$$

one expects a linear evolution of $\mu_{0}^{*}$ with $1 / P^{*}$, with a slope proportional to $C$ and the constant ratio $a^{2} / f_{0}$. Fig. 5

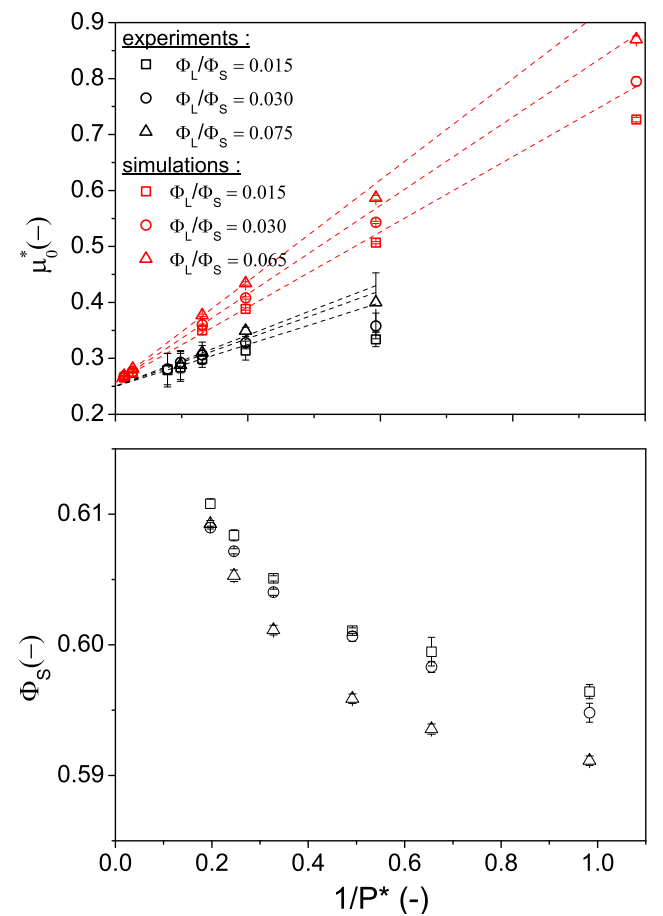

Figure 5. Numerical (open symbols) and experimental (filled symbols) values of $\mu_{0}^{*}$ and $\Phi_{S}$ versus $1 / P^{*}$ for different values of $\Phi_{L} / \Phi_{S}, \eta=550 \mathrm{mPa}$.s. Linear approximation of $\mu_{0}^{*}$ from equation 2 given in dashed lines.

exhibit linear predictions of $\mu_{0}^{*}$ with the values of cohesion given in Table 2, those predictions remain good with less than $5 \%$ relative difference with actual measurements when $1 / P^{*}<1$.

Assuming capillary forces introduce negligible contribution to shear stress, cohesion in granular media can also be approximated theoretically $[3,4]$ as :

$$
C=\mu_{d}^{*} \frac{f_{0}}{a^{2}} \frac{\Phi_{S} z}{\pi}
$$

In the whole range of $1 / P^{*}$, the simulations show that the variations of the product $\Phi_{S} z$ are small (10\% at most), while the cohesion estimated from equation 1 can vary up to $30 \%$ in simulations and up to $40 \%$ in experiments. It is likely that neglecting the contribution of capillary forces to shear stress, when the cohesive effect is high $1 / P^{*}>1$, is too strong an assumption.

Table 2. Values of cohesion $C$ in Pa. As deduced from experiments $\left(C_{\text {exp }}\right)$, simulations $\left(C_{n u m}\right)$ and approximation (eq. $\left.3, C_{\text {app }}\right)$.

\begin{tabular}{llll}
\hline$\Phi_{L} / \Phi_{S}$ & $C_{\text {exp }}$ & $C_{\text {num }}$ & $C_{a p p}$ \\
\hline 0.003 & & 76.7 & 91.2 \\
0.007 & & 77.2 & 93 \\
0.015 & 42 & 77.4 & 95.2 \\
0.030 & 49 & 94.7 & 97.5 \\
0.060 & & 108.2 & 100 \\
0.075 & 50 & 126.2 & 103.8 \\
\hline
\end{tabular}




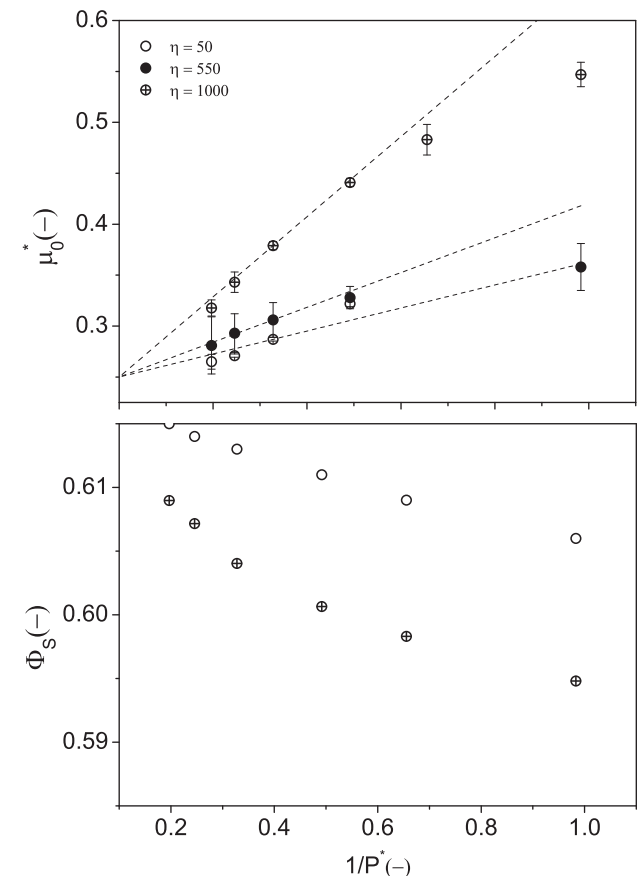

Figure 6. experimental values of $\mu_{0}^{*}$ and $\Phi_{S}$ versus $1 / P^{*}$ for different values of $\eta, \Phi_{L} / \Phi_{S}=0.03$. Linear approximations of $\mu_{0}^{*}$ from equation 2 are given by the dasehd lines.

\subsection{Quasistatic regime : Viscosity effect}

Fig. 6 shows the experimental value of $\mu_{0}^{*}$ and $\Phi_{S}$ versus $1 / P^{*}$ for different values of viscosity $\eta$. As remarked above (Sec. 3.2), the linear variation of $\mu_{0}^{*}$ with $1 / P^{*}$ shows the applicability of a Mohr-Coulomb criterion of plastic flow for $1 / P^{*}<1$.

Identified cohesion values appear to vary with the viscosity of the liquid, ranging from 33Pa for $\eta=50 \mathrm{mPa} . \mathrm{s}$ up to $116 \mathrm{~Pa}$ for $\eta=1000 \mathrm{mPa}$.s. A strong influence of the viscosity on friction coefficient $\mu_{0}^{*}$ is also observed in the cohesion dominated regime, when $1 / P^{*}>1$. Solid fraction $\Phi_{S}$, on the other hand, is not sensitive to $\eta$ variations. While viscous forces through liquid bridges are negligible at low velocities [9], one may speculate that a higher viscosity affects the rate with which liquid is transported between different meniscii in the contact network, leading to more heterogeneous liquid distributions. In turn, such heterogeneities, possibly involving complex structures merging independent meniscii together (as apparent in Fig. 7, obtained by X-ray microtomography on one of the experimental samples) could explain the unexpected increase of $\mu_{0}^{*}$ with $\eta$.

\section{Conclusion}

While the effects of $I, P^{*}$ and $\Phi_{L} / \Phi_{S}$, which are observed in the simulations are satisfactorily understood, the unex- pected influence of the liquid viscosity could be attributed to inhomogeneous distributions of the liquid content in the sheared sample. Such inhomogeneities might affect the rheological properties (and possibly explain the stronger $I$ dependence of experimental data, compared to numerical ones). More systematic microstructural studies using $\mathrm{X}$-ray microtomography are planned to investigate these effects.

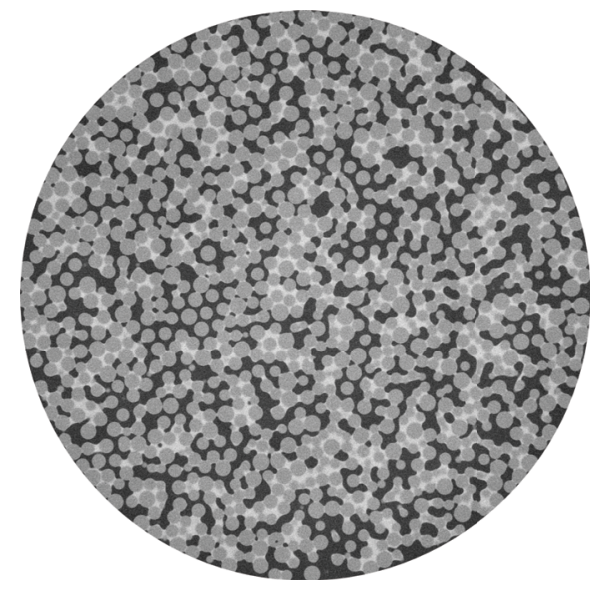

Figure 7. Microtomography image : slice of a sample, $\Phi_{L} / \Phi_{S}=$ 0.075 and $\eta=1000 \mathrm{mPa}$.s.

\section{References}

[1] A. Fall, G. Ovarlez, D. Hautemayou, C. Mézière, J.-N Roux, F. Chevoir, J. of Rheol. 59, 2015

[2] A. Fall, M. Badetti, G. Ovarlez, F. Chevoir, J.-N Roux, these proceedings, 2016

[3] S. Khamseh, J.-N. Roux, F. Chevoir, Phys. Rev. E 92, 2015

[4] V. Richefeu, M.S. El Youssoufi, F. Radjaï, Phys. Rev. E 73, 2006

[5] F.A. Gilabert, J.-N. Roux, A. Castellanos, Phys. Rev. E75

[6] M. Scheel, R. Seemann, M. Brinkmann, M. Di Michiel, A. Sheppard, S. Herminghaus, J.of Phys.:Cond.Matt 20, 2008

[7] N. Mitarai, F. Nori, J.of Phys.:Cond.Matt, 2006

[8] P.C.F. Moller, D. Bonn, EuroPhys. Letters 80, 2008

[9] O. Pitois, P. Moucheront, C; Chateau, J. Coll. Interf. Science 231, 2000

[10] F. Boyer, E. Guazzelli, O. Pouliquen, Phys. Rev. Letter 107, 2011

[11] H. Flyvbjerg, H. G. Petersen, J. Chem. Phys. 91, 1989 